J. Urol. Urogynäkol. AT 2020 • 27:33-37

https://doi.org/10.1007/s41972-020-00101-x

Online publiziert: 16. März 2020

(c) Der/die Autor(en) 2020

\section{Birgit Heller}

Institut für Religionswissenschaft, Universität Wien, Wien, Österreich

\title{
Warum Unreinheit stigmatisiert wird
}

\author{
Blasen-, Darm- und Sexualstörungen aus \\ kulturell-religiöser Perspektive
}

\section{Einleitung}

Der menschliche Körper ist nicht nur eine biologische Gegebenheit, sondern zugleich ein hervorragender Ort kultureller Konstruktion. Aus kulturell-religiösen Perspektiven fallen Phänomene wie Blasen-, Darm- und Sexualstörungen v. a. unter das Stichwort Unreinheit. Unreinheit und Reinheit sind nicht voneinander $\mathrm{zu}$ trennen, sondern bedingen einander. Reinheit ist dabei nicht einfach als ein Synonym für Sauberkeit zu verstehen, sondern bezeichnet weitaus komplexere Sachverhalte. Der Begriff rein stammt von germanisch ${ }^{\star}$ hraini „unvermischt, frei von Schmutz, fehlerlos" und ist verwandt mit griechisch $\kappa \rho เ v \varepsilon เ v$,scheiden, trennen, sichten“" [1]. Reinheit ist daher in seiner Grundbedeutung das Ergebnis eines Vorgangs des Scheidens und Absonderns.

Vorstellungen von Reinheit bzw. Unreinheit tauchen in vielen unterschiedlichen Kontexten auf: von Politik (kulturelle Homogenie, „Rassenhygiene“) über Kunst (Stilreinheit), Psychologie (reine Persönlichkeitstypen), Biologie (Reinrassigkeit), Medizin (Hygiene), Ernährung (Reinheitsgesetz für Bier; Vegetarismus) bis hin zur Werbung (z.B. Waschmittelwerbung). (Un-)Reinheitsvorstellungen, -vorschriften und -riten finden sich quer durch die Kultur- und Religionsgeschichte: In indigenen Kulturen, in den ältesten Schriftkulturen, aber auch in den großen religiösen Traditionen der Gegenwart sind sie verbreitet $[2,3]$.

\section{Zur Funktion von Reinheitsvor- stellungen}

Die Kulturanthropologin Mary Douglas veröffentlichte im Jahr 1966 eine vielzitierte und einflussreiche Studie namens „Purity and danger“ („Reinheit und Gefährdung“, [4]). Sie versammelte und interpretierte darin Einsichten, die sie im Kontext von Feldforschungen zu verschiedensten indigenen Völkern gewann. Bis heute beliebt ist ein Erklärungsansatz für Reinheitsvorschriften, den Douglas als medizinischen Materialismus bezeichnete. Damit gemeint ist die Auffassung, dass alle Reinheitsvorschriften auf Hygienevorstellungen basieren bzw. dem Zweck der Hygiene dienen. Dass Reinheit jedoch nicht zwangsläufig mit Sauberkeit identisch ist, zeigt das Beispiel des indischen Flusses Ganges. Dieser Fluss, der von Hindus als Göttin Gangā verehrt wird, gilt als Inbegriff der Reinheit, obwohl er in westlichen Medien wegen der hohen Verschmutzung als tödliche Kloake bezeichnet wird [5].

Unreinheit und Reinheit sind nach Mary Douglas primär Ordnungskategorien, Nebenprodukte einer soziokulturellen Ordnung - um Unreinheitsvorstellungen einer Kultur zu verstehen, ist es daher nötig, die ihnen zugrunde liegenden Ordnungsvorstellungen zu untersuchen. (Un-)Reinheitsvorschriften stehen im Gesamtkontext jener Gefahren, die in einem Universum möglich sind: Vorstellungen vom Trennen, Reinigen, Abgrenzen und Bestrafen von Überschreitungen haben v. a. die Funktion, eine ungeordnete Erfahrung zu systematisie- ren. Dadurch, dass die Unterschiede zwischen innen und außen, oben und unten, männlich und weiblich, rein und unrein betont werden, kann Ordnung geschaffen werden. Kultur vermittelt Grundkategorien der Ordnung, und Schmutz ist nach Douglas etwas, das „fehl am Platz“ ist: „Unsauberes oder Schmutz ist das, was nicht dazugehören darf, wenn ein Muster Bestand haben soll." Schmutz ist also eine relative Kategorie, die von einem Ordnungssystem abhängt:

„Wo es Schmutz gibt, gibt es auch ein System. Schmutz ist das Nebenprodukt eines systematischen Ordnens und Klassifizierens von Sachen, und zwar deshalb, weil Ordnen das Verwerfen ungeeigneter Elemente einschließt. Schmutz ist eine Reaktion, die alle Gegenstände und Vorstellungen verdammt, die die gängigen Klassifikationen durcheinanderbringen oder in Frage stellen können“" [4].

Schmutz, Zweideutigkeiten, Anomalien sind Symbole der Unordnung und rufen Angst hervor, aber auch Reflexionen über die Beziehung zwischen Ordnung und Unordnung, über Sein und Nichtsein, Leben und Tod. Unreinheitsvorstellungen und die jeweiligen Sanktionen für Unreinheit sind Instrumente, die die Konformität stärken und die Ordnung stabilisieren. Gemeinschaften gewinnen ihre Stabilität, indem die von der Norm abweichende Realität ausgegrenzt wird.

Die Erkenntnisse und Thesen von Mary Douglas sind bis heute aktuell. Die Kategorien Reinheit und Unreinheit bringen einen kulturell-religiösen Wertekosmos zum Ausdruck. Sie schaffen 


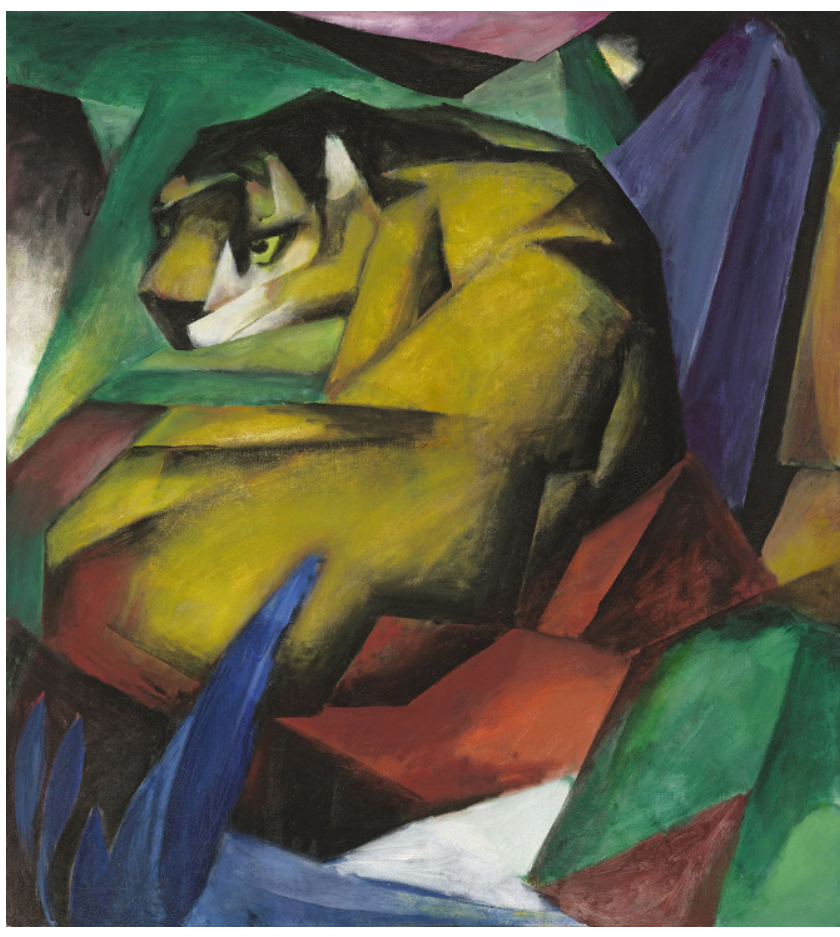

Abb. $1<$ Franz Marc: Tiger. (Aus: https://www. lenbachhaus. de/entdecken/ sammlungonline/detail/tiger30019617; https:// creativecommons. org/licenses/by-sa/ 4.0/deed.de)

Ordnung durch Trennen und Abgrenzen. Reinheit repräsentiert Ordnung, Unreinheit hingegen Unordnung, Chaos und Destruktion. Unreinheit bzw. Reinheit sind zentrale und einflussreiche kulturell-religiöse Kategorien, die dazu dienen, eine bestimmte Ordnung $\mathrm{zu}$ schaffen und aufrechtzuerhalten. Dabei überschneiden sich symbolische, kultische und soziale Ordnungen. Reinheit und Unreinheit sind zunächst symbolische Kategorien, aber zugleich konstituieren sie eine bestimmte soziale Wirklichkeit, indem die Grenzen nach außen und innen geregelt werden: etwa zwischen Gläubigen/Ungläubigen; Klerikern/Laien; Männern/Frauen; Kultfähigen/Nichtkultfähigen; Berührbaren/Unberührbaren usw. Damit werden soziale Hierarchien geschaffen, wobei einzelne Menschen/Gruppen/ Minderheiten, die nicht den Reinheitsnormen entsprechen, stigmatisiert und diskriminiert werden. Allerdings muss betont werden, dass es sich oft um ideale Standards handelt, die nicht immer (strikt) praktisch umgesetzt werden.

\section{Schlüsselrolle: Körper}

Der Körper spielt in den (Un-)Reinheitsdiskursen eine besondere Rolle, weil die Überzeugung universal verbreitet ist, dass insbesondere die Körpergrenzen und Grenzzustände des Körpers bzw. körperliche Grenzüberschreitungen (wie Geburt und Tod, aber auch Sexualität) von Unordnung bedroht sind. Körperliche Grenzzustände und Grenzüberschreitungen gelten als gefährlich und verunreinigend. Es sind v. a. die Körperöffnungen, die im Fokus stehen, und das, was sie passiert: Körperflüssigkeiten, Sexualsekrete, Blut, Urin und Kot und umgekehrt auch die Nahrung. Da der individuelle Körper ein Modell für den Sozialkörper, die Gesellschaft, bildet, werden Körperausscheidungen als Gefahren für die soziale Ordnung betrachtet, die mit der symbolischen (kulturell-religiösen) Ordnung eng verknüpft ist. In der Regel gelten Reinheitsnormen für beide Geschlechter. Häufig werden Frauen jedoch als potenziell unreiner eingestuft. Zum einen, weil sie durch ihre Körperfunktionen (Menstruation, Schwangerschaft, Geburt) regelmäßig in einen Zustand der Unreinheit geraten, und zum anderen, weil sie im dualistischen Denken patriarchal geprägter Gesellschaften generell stärker mit Körper und Sexualität identifiziert werden als Männer. Der weibliche Körper wird als anfälliger für Unreinheit betrachtet und daher stärkeren Reglementierungen unterworfen. Umgekehrt wird Frauen eine exemplarische Bedeutung als Repräsentantinnen von Reinheit zugewiesen, sie symbolisieren die Ordnung des Geschlechterverhältnisses und darüber hinaus die soziale Ordnung. Da die destabilisierenden Kräfte bevorzugt am weiblichen Körper festgemacht werden, stellen die Bedrohungen, die von ihm ausgehen, die patriarchale Ordnung in Frage. Der weibliche Körper ist der Ort, an dem die Grenzen der Gemeinschaft, der normativen Ordnungen und Machtverhältnisse markiert, demonstriert, kontrolliert und stabilisiert werden. Daher gelten Unreinheitsund Unzuchtszuweisungen primär dem weiblichen Verhalten und der Präsentation des weiblichen Körpers.

Reinheitsvorschriften problematisieren und verbieten im Kern physischen Kontakt. Daher grenzen sie ab und variieren kulturell, sind also nicht universal gültig. Anhand verschiedener Beispiele lassen sich Gemeinsamkeiten und Besonderheiten von (Un-)Reinheitsvorstellungen demonstrieren. So wird etwa im Hinduismus der Leichnam als besonders unrein betrachtet, aber auch die Witwe, die eigentlich eine Art lebender Leichnam ist und von der Gemeinschaft ausgestoßen wird, da sie mit ihrem toten Ehemann, der ihr einziger Daseinszweck ist, verbunden bleibt. Verunreinigend wie der Tod ist auch die Geburt, wie der Umgang mit der Wöchnerin illustriert, die z. B. in der katholischen und in der orthodoxen Kirche erst nach dem Ritual der Aussegnung wieder als rein betrachtet wurde bzw. wird [6,7]. Der Ritus wurde in der katholischen Kirche bis 1960 praktiziert, in der Orthodoxie bis heute.

Am Beispiel der Menstruation lassen sich Einstellungen zu sog. unreinen Körperausscheidungen paradigmatisch illustrieren. Menstruation ist das Paradebeispiel eines Bluttabus und gilt als gefährlich, weil sie nicht kontrollierbar ist und mit Tod (erfolglose Empfängnis) verknüpft wird [8]. Menstruation gilt als Beispiel für eine symbolische Anomalie [4]. Sie unterscheidet sich von anderen Formen des Blutens, weil sie nicht kontrollierbar ist. In dieser Hinsicht 
ist sie der Harn- und Stuhlinkontinenz vergleichbar. Was nicht kontrollierbar ist und gesetzte Grenzen überschreitet, gilt als gefährlich für die soziokulturelle Ordnung. Nicht in allen Gesellschaften ist die Menstruation mit Unreinheit verknüpft, d.h. sie ist nicht universal negativ konnotiert [9]. Sie wird jedoch in den großen religiösen Traditionen der Gegenwart, im Judentum, Islam, teilweise im Christentum, in vedischhinduistischen Traditionen, aber auch im Buddhismus, als unrein betrachtet.

Besonders illustrativ sind die jüdischen Reinheitsvorschriften, die Menstruation betreffend. Grundgelegt im Talmudtraktat Nidda besitzen sie bis heute im Judentum große Relevanz. Eine Frau gilt während ihrer Menstruation und nach der Geburt als Nidda und muss eine Vielzahl von Regeln einhalten, um die Familienreinheit zu gewährleisten. Der Begriff Nidda (etymologisch „absondern“) entwickelt sich bereits innerbiblisch $\mathrm{zu}$ einer Grenzkategorie im Sinn der Kontamination und Absonderung, die Frauen mit Unreinheit in Verbindung bringt $[10,11]$. Das biblische Buch Levitikus enthält Reinheitsvorschriften, die die Reinheit Israels garantieren sollen. Prinzipiell können sich zwar beide Geschlechter verunreinigen, aber die verunreinigende Kraft, die von der physiologischen Menstruation ausgeht, wird mit pathologischen Ausflüssen beim Mann parallelisiert. Im rabbinischen Judentum erhält die weibliche Unreinheit eine dominante Bedeutung [12]. Der Talmudtraktat Nidda rückt die Familienreinheit verknüpft mit sexueller Enthaltsamkeit ins Zentrum. Für die Reinheit der Familie werden primär Frauen verantwortlich gemacht. Die Nidda-Zeitspanne wird erweitert von 7 (im biblischen Buch Levitikus) auf 14 Tage: die 7 Tage der Periode und die sogenannten 7 weißen Tage, danach müssen Frauen die Mikwe, das rituelle Tauchbad, besuchen, um wieder als rein zu gelten. Diese Vorschriften bestimmen bis heute das Sexualleben von traditionellen jüdischen Ehepaaren: Körperliche Berührungen sind verboten. Frauen sind überdies zumindest im orthodoxen Judentum aus dem öffentlichen und religiösen Gemeindeleben

J. Urol. Urogynäkol. AT 2020 · 27:33-37 https://doi.org/10.1007/s41972-020-00101-x (c) Der/die Autor(en) 2020

\section{B. Heller \\ Warum Unreinheit stigmatisiert wird. Blasen-, Darm- und Sexualstörungen aus kulturell-religiöser Perspektive}

\section{Zusammenfassung}

(Un-)Reinheitsvorstellungen, -vorschriften und -riten finden sich quer durch die Kulturund Religionsgeschichte. Vorstellungen vom Trennen, Reinigen, Abgrenzen und Bestrafen von Überschreitungen haben v. a. die Funktion, eine spezifische Ordnung zu schaffen und aufrechtzuerhalten. Reinheit symbolisiert Ordnung. Umgekehrt sind Schmutz,

Zweideutigkeiten und Anomalien Symbole der Unordnung und rufen Angst hervor. (Un-)Reinheitsvorschriften dienen der Stabilisierung von Ordnung, zugleich konstituieren sie eine bestimmte soziale Wirklichkeit, indem die Grenzen nach außen und innen geregelt werden (Gläubige/Ungläubige; Kleriker/Laien; Männer/Frauen; Berührbare/Unberührbare usw.). Damit werden soziale Hierarchien geschaffen, wobei einzelne Menschen/Gruppen/Minderheiten, die nicht den Reinheitsnormen entsprechen, stigmatisiert und diskriminiert werden. Allerdings handelt es sich oft um ideale Standards, die nicht immer praktisch umgesetzt werden. Der Körper spielt eine besondere Rolle, weil die Überzeugung universal verbreitet ist, dass insbesondere die Körpergrenzen von Unordnung bedroht sind. Es sind v. a. die Körperöffnungen, die im Fokus stehen, und das, was sie passiert: Körperflüssigkeiten, Sexualsekrete, Blut, Urin und Kot und umgekehrt auch die Nahrung. In vielen Gesellschaften wird der weibliche Körper als anfälliger für Unreinheit betrachtet als der männliche. Da der individuelle Körper ein Modell für den Sozialkörper, die Gesellschaft, bildet, gelten Körperausscheidungen als Gefahren für die soziale Ordnung, die mit der symbolischen (kulturell-religiösen) Ordnung eng verknüpft ist.

\section{Schlüsselwörter}

Abgrenzung, Anomalie · Ordnung · Körperöffnungen · Geschlecht · Diskriminierung

\section{Why impurity is stigmatized. Bladder, bowel and sexual disorders from a cultural-religious perspective}

\section{Abstract}

Ideas, regulations and rituals of (im)purity can be found across the history of culture and religion. The idea of separating, purifying, delimiting and punishing of transgressions has the primary function of creating and maintaining a specific order. Purity symbolizes order. Conversely, dirt, ambiguities and anomalies are symbols of disorder and cause fear. Regulations of (im)purity serve to stabilize order, at the same time they constitute a certain social reality by regulating the borders internally and externally (believers/unbelievers; clerics/laity; men/women; touchables/untouchables, etc.). This creates social hierarchies, whereby individual people/groups/minorities who do not meet purity standards are stigmatized and discriminated against. However, these are often ideal standards that are not always implemented in practice. The body plays a special role because the conviction is universal that especially the body boundaries are at risk of disorder. It is primarily the body orifices that are in focus and what passes through them: body fluids, sexual secretions, blood, urine and feces, but also food. In many societies, the female body is regarded to be more affected by impurity than the male body. Since the individual body is a model for the social body that forms society, excretions of the body are considered to be dangerous to the social order, which is closely linked to the symbolic (cultural-religious) order.

\section{Keywords}

Separation, Anomaly · Order · Body orifices . Sex - Discrimination ausgeschlossen. Tempel- bzw. Moscheebesuch sind menstruierenden Frauen auch im traditionellen Hinduismus und im Islam untersagt.

\section{(Un-)Reinheit in säkularen Gesellschaften}

(Un-)Reinheitsvorstellungen sind alles andere als abstruse Relikte rückständiger Kulturen und religiöser Überlieferungen. 
Körpertabus sind nicht auf Religionen beschränkt, sondern auch in säkularen Gesellschaften anzutreffen. Moderne Gesellschaften haben beispielsweise extreme Hygienevorstellungen entwickelt, die mit einem Überangebot an Putzmitteln und Körperpflegeprodukten in Reinlichkeitswahn und Sterilitätsidealen ausarten. Möglicherweise erinnern Körperausscheidungen den modernen Menschen zu sehr an die "Unordnung“ der Sterblichkeit, die er um jeden Preis überwinden möchte? Dem Ekel vor der Hinfälligkeit und dem Tod wird das Ideal eines reinen, unverletzlichen, perfekten Körpers entgegengesetzt. Auch in säkularen Gesellschaften führen Körperausscheidungen $\mathrm{zu}$ sozialen Ausschlüssen und zwingen zur Konformität in Verhaltensmustern. In der Kunst taucht bereits zu Beginn des 20. Jahrhunderts eine Rebellion gegen die Ordnungszwänge der zeitgenössischen Reinheitsbestrebungen auf, und die Darstellung der Körperausscheidungen dient der Gesellschaftskritik [13]. Franz Marc deutete sein eigenes künstlerisches Schaffen 1915 noch mit der Idee der „reinen Kunst“. Seine Bilder zeigen Tiere, die sich durch die zunehmende Abstraktion ihrer Körper auf geometrische Formen und eine Verengung der Farbgebung auszeichnen. Diesem Bemühen um Reinheit durch Abstraktion tritt eine vehemente Zurschaustellung schmutziger Realitäten in der modernen Malerei entgegen (vgl. dazu die Bilder von Franz Marc: Tiger, s. Abb. 1, versus George Grosz: Die Niederkunft, siehe http://bit.ly/2RVymq7). Die Macht, den Verhaltenscode zu bestimmen, ist letztlich nur so groß wie der Respekt der Menschen für den jeweiligen Code. Einstellungen und Verhaltensmuster können sich verändern, wenn deutlich gemacht wird, welche Überzeugungen ihnen zugrunde liegen. Vorstellungen von (Un-)Reinheit sind kulturelle Konstrukte, keine natürlichen Gegebenheiten.

\section{Fazit für die Praxis}

- (Un-)Reinheitsvorstellungen sind nicht nur in sog. rückständigen Kulturen und religiösen Überlieferungen verbreitet, sondern haben auch in modernen Gesellschaften eine einflussreiche Bedeutung.

- Die Stigmatisierung und Diskriminierung von sog. unreinen Menschen ist nicht allein als Konsequenz von Sauberkeitsnormen zu erklären.

- Menschen, die von Blasen-, Darmund Sexualstörungen betroffen sind, stören potenziell die soziale Ordnung. Hinter dem peinlichen Verschweigen, der Vermeidung und Ausgrenzung der Betroffenen steckt die Angst vor dem Kontrollverlust, der Unordnung, letztlich vor Hinfälligkeit und Tod.

- Diese Einstellungen können hinterfragt werden, wenn deutlich gemacht wird, dass es sich um kulturelle Konstrukte handelt.

\section{Korrespondenzadresse}

Ao. Univ.-Prof.in Mag.a Dr.in Birgit Heller Institut für Religionswissenschaft, Universität Wien

Schenkenstraße 8-10, 1010 Wien, Österreich birgit.heller@univie.ac.at

Ao. Univ.-Prof.in Mag.a Dr.in Birgit Heller Stellvertretende Institutsvorständin. Präsidentin der Österreichischen Gesellschaft für Religionswissenschaft.

Funding. Open access funding provided by University of Vienna.

\section{Einhaltung ethischer Richtlinien}

Interessenkonflikt. B. Heller gibt an, dass kein Interessenkonflikt besteht.

Für diesen Beitrag wurden von der Autorin keine Studien an Menschen oder Tieren durchgeführt. Für die aufgeführten Studien gelten die jeweils dort angegebenen ethischen Richtlinien.

Open Access Dieser Artikel wird unter der Creative Commons Namensnennung 4.0 International Lizenz veröffentlicht, welche die Nutzung, Vervielfältigung, Bearbeitung, Verbreitung und Wiedergabe in jeglichem Medium und Format erlaubt, sofern Sie den/die ursprünglichen Autor(en) und die Quelle ordnungsgemäß nennen, einen Link zur Creative Commons Lizenz beifügen und angeben, ob Änderungen vorgenommen wurden.

Die in diesem Artikel enthaltenen Bilder und sonstiges Drittmaterial unterliegen ebenfalls der genannten Creative Commons Lizenz, sofern sich aus der Abbildungslegende nichts anderes ergibt. Sofern das betreffende Material nicht unter der genannten Creative Commons Lizenz steht und die betreffende Handlung nicht nach gesetzlichen Vorschriften erlaubt ist, ist für die oben aufgeführten Weiterverwendungen des Ma- terials die Einwilligung des jeweiligen Rechteinhabers einzuholen.

Weitere Details zur Lizenz entnehmen Sie bitte der Lizenzinformation auf http://creativecommons.org/ licenses/by/4.0/deed.de.

\section{Literatur}

1. Pfeifer W (Hrsg) (1989) Etymologisches Wörterbuch des Deutschen 3. Q-Z, Akad.-Verl, Berlin

2. Malinar A, Vöhler M (Hrsg) (2009) Un/Reinheit Konzepte und Praktiken im Kulturvergleich. Fink München

3. Burschel P, Marx Ch (Hrsg) (2011) Reinheit. Veröffentlichungen des Instituts für Historische Anthropologie, Bd. 12. Böhlau, Wien, Köln, Weimar

4. Douglas M (1988) Reinheit und Gefährdung. Eine Studie zu Verunreinigung und Tabu [= Purity and Danger, dt]. Suhrkamp, Frankfurta.M.

5. http://www.weltderwunder.de/artikel/indiensheiliger-fluss-wo-ein-schluck-wasser-toedlichist [Zugriffam: 20.01.2020]

6. Roll SK (2003) The old rite of the churching of women after childbirth. In: De Troyer K (Hrsg) Wholly woman, holy blood. A feminist critique of purity and impurity (studies in antiquity and christianity). Trinity Press International, Harrisburg, London, New York, S 117-142

7. Synek E (2006) „Wer aber nicht völlig rein ist an Seele und Leib ..." Reinheitstabus im Orthodoxen Kirchenrecht. Edition Roman Kovar, Egling

8. Meyer ML (2005) Thicker than water. The origins of blood as symbol and ritual. Routledge, New York

9. Buckley Th, Gottlieb A (1988) A critical appraisal of theories of menstrual symbolism. In: Buckley Th, Gottlieb A (Hrsg) Blood magic. The anthropology of menstruation. Univ. of Calif. Press, Berkeley, Los Angeles, London, S3-50

10. Eberle-Küster D (2008) Körper und Geschlecht. Studien zur Anthropologie von Leviticus 12 und 15 (WMANT 121). Neukirchener Verlag, NeukirchenVluyn

11. De Troyer K (2003) A threat to holiness or toward (another) holiness? In: De Troyer K (Hrsg) Wholly woman, holy blood. A feminist critique of purity and impurity (studies in antiquity and christianity). Trinity Press International, Harrisburg, London, New York, S45-64

12. Meachem T (1999) An abbreviated history of the development of the jewish menstrual laws. In: Wasserfall RR (Hrsg) Women and water. Menstruation in jewish life and law, brandeis. Univ. Press, Hanover, New Hampshire, London, S23-39

13. Fayet R (2009) Vom „Nirwana der Reinheit" zur Fäkaliendose. Un/Reinheit und Post/moderne. In Malinar A, Vöhler M (Hrsg) Un/Reinheit. Konzepte und Praktiken im Kulturvergleich. Fink, München, S251-277

Hinweis des Verlags. Der Verlag bleibt in Hinblick auf geografische Zuordnungen und Gebietsbezeichnungen in veröffentlichten Karten und Institutsadressen neutral. 
Hier steht eine Anzeige.

\section{曾 Springer}

